Res Pública Revista de Historia de las Ideas Políticas

ISSN: $1131-558 \mathrm{X}$

https://dx.doi.org/10.5209/rpub.75858

\title{
Marx, ciencia de la contingencia
}

\author{
Alejo Stark ${ }^{*}$
}

Recibido: 04-05-2021 / Aceptado: 20-09-2021

Resumen. En su libro On the Nature of Marx's Things Jacques Lezra hereda otro Marx, y otro materialismo. Un materialismo aleatorio, un materialismo de la contingencia dinámica de Marx y sus "cosas". Tal "corriente subterránea" del materialismo aleatorio es excavada por Lezra en su desvío por las cartas, cuadernos y notas "privadas" de un joven Marx que trabajaba en su tesis de doctorado. Siguiendo el hilo necrofilológico de Lezra, que se topa con Lucrecio y sus "cosas," encontramos que, paralelamente, Marx también busca un concepto de ciencia en su tesis: lo que tentativamente nombramos ciencia de la contingencia. Ciencia, no ya disciplinaria, que abre la posibilidad de una alianza con el materialismo performativo de Karen Barad que desplaza el humanismo de los "nuevos materialismos" desde una reflexión critica que piensa a la par de otra ciencia de la contingencia, también de cierta herencia lucreciana, con la cual Marx no llegó a pensar: la mecánica cuántica. Palabras clave: Jacques Lezra; Marx; materialismo aleatorio; ciencia; Lucrecio.

\section{[en] Marx, Science of Contingency}

Abstract. In his book On the Nature of Marx's Things Jacques Lezra inherits another Marx and another materialism. It is an aleatory materialism: a materialism of the dynamic contingency of Marx and his "things". This "subterranean current" of aleatory materialism is excavated by Lezra in his swerve through the letters, notebooks, and "private notes" of a young Marx working on his doctorate thesis. Following Lezra's necrophilological thread -which encounters Lucretius and his "things"we find that, in a parallel fashion, Marx is also searching for a concept of science in his dissertation. This is what here is tentatively called a science of contingency. This science - though not in the usual disciplinary sense- opens the possibility of an alliance with Karen Barad's performative materialism that displaces the humanism of the "new materialisms" by thinking alongside another science of contingency, also of a certain Lucretian inheritance, and which Marx did not get to think: quantum mechanics.

Keywords: Jacques Lezra; Marx; Aleatory Materialism; Science; Lucretius.

Sumario. 1. Contingencia. 2. Ciencia. 3. Alianzas. Bibliografía

Cómo citar: Stark, A. (2022). Marx, ciencia de la contingencia. Res Pública. Revista de Historia de las Ideas Politicas, 25(2), 31-39.

Leyendo el último libro de Lezra -titulado On the nature of Marx's things: Translation as Necrophilology-nos encontramos con una escritura en marcha ${ }^{1}$. Una escritura que nunca se siente en casa y se mueve como un pensamiento viajero que avanza por desvíos (declinatio) y por saltos (per saltum). Escritura adversa a las ataduras de la identidad -pero no de la mediación- y de aquellos materialismos que, en su absolutización de lo uno, remiten a un fetichismo teológico humanista. Escritura del exilio que busca una cierta cercanía -al tomar distancia- con la "ciencia melancólica" de Adorno que se encuentra en los aforismos de Minima Moralia. Y también escritura que avanza por medio de fragmentos -aunque no aforísticos-que no suman a una totalidad pero sí van tomando consistencia en un complejo entramado de relaciones (leído como connnexio y textura). Nos encontramos así frente a un problema formidable: ¿desde dónde comenzar a dar cuenta de tal complejidad del texto de Lezra?

Como el título del libro indica, a esta escritura se la puede seguir por medio del hilo de un nombre propio, el de Marx, y de sus "cosas". Este título nos remite a otro, el del poeta-filósofo Lucrecio, De la naturaleza de las cosas, al que Lezra acude para reactivar una tendencia del pensamiento de Marx que habría sido reprimida. Lezra hereda otro Marx, y por lo tanto otro materialismo, al excavar lo que el filósofo comunista Louis Althusser, en un fragmento tardío, llamó la "corriente subterránea" del "materialismo del encuentro" que él mismo leyó, por

\footnotetext{
University of Michigan

alejo@umich.edu

1 Tal libro no ha sido traducido todavía al castellano. Por lo tanto, en lo que sigue citamos el trabajo de Jacques Lezra directamente en inglés sin traducción.
} 
un lado, en la lluvia paralela de átomos de Epicuro de la que escribe Lucrecio como también, por otro lado, en el paralelismo de los atributos infinitos de la naturaleza de Spinoza ${ }^{2}$. El fragmento de Althusser comienza in medias res, en el "medio" de las cosas. "Llueve". Flujo de materia en el vacío que con una simple declinación o clinamen de su trayectoria paralela engendra un encuentro. Así "comienza" el pensador materialista: en el ir y venir de las cosas, en movimiento, sin origen ni telos. En su encuentro con el mundo, sus nombres, sus cosas, el "filósofo materialista" que evoca Althusser, y también la escritura lezreana, es aquel que "toma el tren en marcha...[s]in saber de dónde viene (origen) ni a dónde va (fin)... [y] se baja en marcha, en un pequeño poblacho en torno a una estación ridícula"3. Un materialismo del encuentro, de lo aleatorio, de la contingencia ${ }^{4}$.

Herencia, en el sentido derrideano, implica necesariamente criticar y filtrar ${ }^{5}$ - como una arqueóloga que criba la tierra o una astrónoma que difracta la luz estelar. Desde las primeras páginas del libro, Lezra caracteriza su proyecto como un ejercicio crítico-político. Por lo tanto, la promesa crítica-política del texto y sus fragmentos pasarían, en primer lugar, por heredar el legado materialista de Marx, en particular el lucreciano y el spinoziano, que habría sido rechazado ("disavowed") por cierta tradición dialéctica como, por ejemplo, la de Engels y su Dialéctica de la naturaleza. Pero también, y en segundo lugar, por esbozar los modos en los cuales tal ejercicio crítico nos dejaría no solo "pensar" sino también "modificar" o "desplazar" lo que él llama las "catástrofes del capital".

Siguiendo el hilo del trabajo necrofilológico de Lezra, la promesa de lo que sigue es pensar lo que provisionalmente- podríamos llamar una ciencia de la contingencia en Marx. Ciencia, no ya en un sentido estrictamente disciplinario, que abre la posibilidad de una alianza con el trabajo del materialismo performativo de Karen Barad que, a diferencia del materialismo negativo de Quentin Meillasoux con el cual se entrevera Lezra, enfatiza la primacía de la relación (o mediación), la iterabilidad, y la indeterminación en sus indagaciones en torno a otra ciencia de la contingencia, también de herencia lucreciana, que Marx no llegó a pensar: la mecánica cuántica.

\section{Contingencia}

Es en las cartas, cuadernos y notas "privadas" de un joven Marx que con casi 20 años trabajaba en su tesis de doctorado sobre la diferencia entre las filosofías de la naturaleza de Epicuro y Demócrito con Bruno Bauer donde Lezra lee el encuentro de Marx con Lucrecio. Es ahí donde la lectura lezreana, en el "dinamismo contingente" de la poesía-filosofía lucreciana que también leerá, mediado por una cierta traducción, las dinámicas

L. Althusser, Para un materialismo aleatorio, trad. P Fernández Liria y G. González, Madrid, Arena Libros, 2002.

Ibidem, p. 9.

Ibidem, p. 31.

J. Derrida, Espectros de Marx. El estado de la deuda, el trabajo del duelo y la nueva internacional, Madrid, Editorial Trotta, 1998, p. 30. contingentes de la forma-valor asumida por el producto de trabajo. Aunque en rigor no trabajaremos precisamente esta relación, Lezra trata de sostener un hilo -o, más bien, un complejo entramado de relaciones entre cosas, conceptos, y nombres, ...-que vincula este momento de cierta acumulación originaria en el corpus de Marx con la ciencia-crítica que encontramos en El capital ${ }^{6}$. Esto es lo que elaboraremos, seguramente de una manera un poco precipitada, y aquello que nombrábamos como ciencia de la contingencia.

La apuesta de Lezra es la siguiente: mostrar cómo cierto desvío de Marx por Lucrecio desestabiliza las identidades de conceptos-cosas establecidas por cierta teoría del valor en torno al problema del fetichismo (que considera el valor como externo a la circulación de la mercancía) y, en contraste, a la crematística (que considera el valor de una mercancía en relación a su circulación $)^{7}$. Es decir, Lezra sigue el hilo que ata la lectura de Marx de Lucrecio y como ella es traducida al plano de la teoría del valor. Pero tal herencia funciona en un registro o patrón ("pattern"), en el cual Marx imprime cierta forma a otros modos de pensamiento ${ }^{8}$. En todo caso, lo que está en juego para Lezra es el "uso" que Marx le da a Lucrecio.

"It goes without saying that but little use can be made of Lucretius". Con esta frase Marx comienza el cuarto capítulo de sus Cuadernos sobre la filosofía epicúrea, con la que Lezra abre el primer capítulo de On the Nature of Marx's Things ${ }^{9}$. Se trata de las cosas de Marx, sus nombres, sus usos y sus constantes traducciones a registros filosóficos, históricos, y también necro-biográficos. El hilo que sostiene este capítulo, hilo-cuerda por donde Lezra camina siguiendo a Marx, se encuentra en una relación paralela a la lectura de Marx de Lucrecio. Marx está fascinado por los saltos mortales de los versos poéticos lucrecianos. Para él, Lucrecio es una suerte de acróbata. Luftspringer es la palabra de Marx que maravilla a Lezra: saltador-aéreo, que en sus frases baila y salta sobre una cuerda elevada sobre el mundo y sus cosas. Esta es la nota "privada" del joven pensador -escrita por y para Marx:

As nature in spring lays herself bare and, as though conscious of victory, displays all her charm, whereas in winter she covers up her shame and nakedness with snow and ice, so Lucretius, fresh, keen, poetic master of the world, differs from Plutarch, who covers his paltry ego [his "small 'I'": sein kleines Ich] with the snow and ice of morality.

\footnotetext{
Comprendiendo que tal "acumulación originaria" es necesariamente aleatoria. Como demuestra Jason Read - siguiendo la lectura de Marx de Althusser como también la de Deleuze y Guattari- la teoría de acumulación primitiva en El capital es una "fundación aleatoria". Cf. J. Read, "Primitive Accumulation: The Aleatory Foundation of Capitalism", Rethinking Marxism, 14,2, 2002, pp. 24-49.

Marcando esa diferencia es cómo Lezra anticipa tal discusión en la introducción de On the Nature of Marx's Things. Esta distinción tiene una larga tradición en la crítica de la economía política marxista. Tal diferencia, por ejemplo, se puede leer en la teoría "circulacionista" del valor de Isaak I. Rubin y, más recientemente, en el trabajo de Moishe Postone. Es curioso que ni Rubin ni Postone aparezcan citados por Lezra en su discusión de la forma valor.

8 J. Lezra, On the Nature of Marx's Things, New York, Fordham University Press, 2018, p. 38-53.

Ibidem, p. 38.
} 
When we see an individual anxiously buttoned-up and clinging into himself, we involuntarily clutch at coat and clasp, make sure that we are still there, as if afraid to lose ourselves. But at the glimpse [Anblick] of an intrepid acrobat we forget ourselves, feel ourselves raised [erhaben] out of our own skins like universal forces [allgemeine Mächte] and breathe more fearlessly. Who is it that feels in the more moral [sittlich] and free state of mind -he who has just come out of Plutarch's classroom, reflecting on how unjust it is that the good should lose with life the fruit of their life, or he who sees eternity fulfilled, hears the bold thundering song of Lucretius? ${ }^{10}$.

Para Lezra, estas líneas de Marx son maravillosas ("marvelous") - "rich and complex, evocative, precise". En ellas nos encontramos con un Marx con su identidad en juego, un Marx dividido, es decir, multiplicado. Un Marx que al afirmar que ningún uso se puede hacer de Lucrecio - "It goes without saying that but little use can be made of Lucretius"- precisamente lo usa para enfrentar a Plutarco y su "pequeño yo" ("small 'I", "paltry ego"). Lezra escribe que tal uso del poeta es una suerte de performance ${ }^{11}$. Marx nos da un Lucrecio acróbata caminando sobre el mundo por una cuerda, maniobra que nos deja medir la distancia de aquel mundo terrenal helado y nevado donde se encuentra Plutarco y su moral. En esa distancia se juegan las cosas de este Marx. Escribe Lezra, "[f]or this early Marx, to read Lucretius is to come across that which elevates-exposes us and threatens us with a loss of self that is correlative to the expansion of our «small I» into the space between the acrobat and the ground, between the world of poetic figures and the wintry ground of things" 12 .

Es precisamente esa distancia -entre el mundo elevado de las figuras poéticas lucrecianas que miran desde allí a la superficie nevada donde se encuentra Plutarco tiritando y sujetando su saco- en la que Marx, el Marx que se escribe a sí mismo, encuentra una suerte de $l i$ bertad ("free state of mind"). ¿Pero es ese el uso que le da Marx a Lucrecio? ¿El de leer en el poeta del clinamen -en tanto su temática y su forma- una suspensión de la necesidad que meramente afirmaría a la contingencia como expresión máxima de un idealismo de la libertad $?^{13}$ Lezra lee cuidadosamente estas notas de Marx y nota que el léxico de tal joven, y el uso que él le da a Lucrecio, es propio de una tradición estética kantiana, pero no reducible a ella.

El "circo" de Lucrecio le ofrece a Marx una vista de la experiencia de libertad de lo sublime a través de una suerte de olvido del mundo terrenal. Lezra resalta que Marx tambalea en estas notas y que, de a momentos, toma distancia de tal lectura de la "libertad" como mera interrupción, como experiencia sublime, que se encuentra al alejarse de lo terrenal y acercarse a las formas

3 Sobre este "uso" de Lucrecio por Marx en el texto de Lezra veáse también S. Villalobos-Ruminott, "La querella de las cosas: Marx, Lucrecio y el desorden del mundo", Oxímora Revista Internacional de Ética y Política, 2020, p. 126.
}

poéticas que sobrevuelan al mundo y sus cosas. Este es precisamente el problema al cual Marx se enfrentó en su tesis: pensar la compleja relación entre el ser y el pensamiento, tarea que Marx se da a pensar por medio de Demócrito y Epicuro y sus respectivas filosofías de la naturaleza.

Marx concluyó su tesis con un capítulo sobre los meteoritos, cuerpos celestes que anuncian cierta confirmación de la teoría de Epicuro - pero que el atomista griego no reconoce ${ }^{14}$. Estas últimas líneas de la Diferencia de la Filosofía de la Naturaleza en Demócrito y Epicuro son anticipadas por un elogio de Lucrecio a Epicuro. En ellas, nuevamente nos encontramos con la compleja relación entre el "cielo" y la "tierra". En la cita de Marx, Lucrecio celebra a Epicuro como el héroe que se enfrenta a los rayos y "estruendos amenazantes" de los dioses y "las religiones del cielo" y que este termina aplastando "bajo sus pies" - recordemos que es Plutarco el que, dice Marx, "arrastra a la filosofía ante el tribunal de la religión"15. La "victoria" de Epicuro por sobre los dioses, escribe Marx, "nos exalta al cielo". Inmediatamente siguiendo la cita de Lucrecio, Marx avanza hacía su conclusión de la gran diferencia entre la filosofía de Epicuro - a quien llama "el mas grande iluminista"- y la de Demócrito.

Por un lado, Epicuro emerge como un proto-Hegel por el cual "la atomística se desarrolla y completa sus contradicciones como ciencia natural de la auto-conciencia", pero este llega a "la suprema consecuencia, es decir, hasta su disolución y su consciente oposición a lo universal". En contraste, para Demócrito "el átomo resulta sólo la expresión general objetiva del estudio empírico de la naturaleza"; el átomo es una "categoría pura y abstracta, una hipótesis, que es el resultado de la experiencia y no su principio energético", y que no llega a "realizarse, así como la investigación real de la naturaleza no es ya determinada por esa experiencia"16. A partir de esta caracterización de Demócrito nos resuena el concepto de ciencia de otro gran iluminista: Kant. Tal es la tesis que elabora Peter Fenves, con la que concuerdo, y que elaboraré en lo que sigue ${ }^{17}$.

En estas líneas nos encontramos con un gran enfrentamiento entre dos conceptos de ciencia. Por un lado, el de Hegel y la Ciencia de la Lógica con su dialéctica de la contradicción que se realiza en la auto-conciencia, y por otro, el de Kant y su idealismo transcendental que provee los fundamentos "puros" para el estudio de la naturaleza llevado a cabo por las ciencias positivas. Es en torno a esta confrontación que Peter Fenves plantea lo que se juega en la tesis doctoral de Marx ${ }^{18}$. A saber, la Diferencia es un Kampfplatz, - un escenario de guerradonde se enfrentan dos conceptos de ciencia y donde Marx escenifica, en una performance dramática en la cual Kant y Hegel "llevan las máscaras" de los dos atomistas clásicos, su toma de posición en torno a los filó-

\footnotetext{
14 K. Marx, Diferencia de la filosofía de la naturaleza en Demócrito y en Epicuro, Madrid, Editorial Ayuso, 1971, pp. 59-60.

17 P. Fenves, "Marx's Thesis on Two Greek Atomists and the Post-Kantian Interpretations", Journal of the History of ldeas, 47, 1986.

18 Ibidem, p. 433-4.
} 
sofos alemanes ${ }^{19}$. Lo que nos interesa de esta lectura de Fenves es, primero, cómo demuestra que "the outcome of the atomists' struggle will determine the function of contradiction and the nature of science, ${ }^{20} \mathrm{y}$, en segundo lugar, que nos deja pensar el rol que juega Lucrecio en tal lucha ("struggle") en torno al concepto de ciencia.

Aunque en rigor es Demócrito la figura que Marx nos presenta como aquel que se preocupa por las leyes físicas (empíricas) del cosmos, es precisamente Epicuro, pensador de la contingencia y de la contradicción que también "desprecia las ciencias positivas" 21 , a quien Marx identifica como el creador "la ciencia de la atomística" debido que este ha "objetivado la contradicción -en el concepto de átomo- entre la esencia y la existencia"22.

Fenves indica que la presentación del concepto de átomo parece estar articulada por la contradictoria unidad dialéctica entre ser y pensamiento. Tal formulación no sorprende, y se podría leer el desenlace de tal dialéctica en la tesis de Marx. Pero como plantea Fenves, si Marx hubiese logrado demostrar que el desarrollo del concepto -concepto de átomo en este caso- puede ser demostrado tanto al nivel histórico como lógico pareciera que la Ciencia hegeliana se confirmaría. Pero como indica Fenves, Marx parece estar incómodo con la $A u$ fhebung especulativa hegeliana. Por ejemplo, Marx evita hablar de la atracción de los átomos en la tesis -un concepto clave para la superación dialéctica del atomismo elaborada por Hegel en la Ciencia de la Lógica y que da paso de la cualidad a la cantidad. En segundo lugar, Marx tampoco parece resolver la contradicción en el concepto de átomo por medio de la Aufhebung especulativa. Aunque, como sí indica Lezra, Marx sigue la lectura de Hegel del declinatio lucreciano, la contradicción epicureana del átomo -la primera determinación del átomo como relación, como punto en una línea recta, y la segunda determinación en su clinamen-, implica una abstracción de la relación. Según Fenves, esta contradicción nos lleva a lo que él llama externalidad y pura materialidad y, por lo tanto, no a una Aufhebung especulativa. Veamos lo que escribe Marx,

Consideremos ahora la consecuencia que inmediatamente se deriva de la desviación [declinatio] del átomo...Pero lo que aquí está contenido, la negación del átomo de toda relación con algo distinto, debe ser realizada y puesta positivamente. Esto sólo puede acontecer si el ser con el cual él se relaciona no es otro que él mismo, es decir, también un átomo, y puesto que es determinado inmediatamente, una pluralidad de átomos. Así el rechazo (Repulsión) de los átomos múltiples es la realización necesaria de la lex atomi, (ley del átomo), como Lucrecio llama a la declinación ${ }^{23}$.

La diferencia entre Marx y Hegel en este punto sería no solamente el hecho de que Marx no recurre al concepto de atracción y por lo tanto no hay reconciliación

Ibidem, p. 434.

Idem.

21 K. Marx, Diferencia de la filosofía de la naturaleza en Demócrito y en Epicuro, op. cit., p. 18.

22 Ibidem, p. 41.

23 Ibidem, pp. 32-33. posible o superadora - si no que para Marx, en cambio, tal desvío por el otro que "no es otro que él mismo" nos devuelve nuevamente a la materia, al átomo. A saber, nos devuelve a la externalidad y "pura" materialidad de la cual habla Fenves.

Es de esa manera que se podría leer el "tercer aspecto" decisivo en el encuentro entre Marx y Lucrecio del que escribe Lezra ${ }^{24}$. Puede ser que el acto de Marx de filtrar a Lucrecio por Hegel nos lleve nuevamente a la materialidad a la que llega Epicuro (pero que no reconoce). Para este Marx, escribe Fenves, "[t]he contradiction between essence and existence, between thought and being, cannot be overcome in the negation of the negation, «the speculative par excellence»" ${ }^{25}$. ¿No se podría leer aquí una instancia de lo que Vittorio Morfino, siguiendo a Althusser, ha caracterizado como el primado del encuentro sobre la forma en Lucrecio y lo que Spinoza propone como demostración de la diferencia entre la explicación científica y la religiosa $?^{26}$ Es decir, que al tratar de mantener la forma, se reprime la materialidad que retorna en ese encuentro. La contingencia radical de toda forma nos desplaza de una filosofía de la forma a una filosofía del encuentro.

Volvamos a la batalla que se da en la tesis de Marx en torno a los meteoritos. La materialidad del cuerpo celeste acecha nuevamente la contradicción de la "ciencia atomista" - la contradicción entre ser y pensamiento que termina por confundir conceptos con objetos sin pensar su relación. Consecuencia que termina por humillar a Epicuro en tanto que éste no puede reconocer el meteorito. La materialidad reprimida retorna sin poder ser reconocida por el atomista. Fenves describe cómo en este momento, Epicuro se aproximó a Plutarco: "[t]he desires to overcome the positive sciences in Epicurus and the justification of religious mystification in Plutarch are, in truth, the same phenomenon". Es también Lucrecio la figura que Marx usó tanto para elogiar, como ya hemos visto, pero también para reírse de Epicuro: la materia, reprimida por el concepto vuelve esta vez con la máscara de la muerte, "Así, la muerte de la naturaleza se convierte en su sustancia inmortal, y con razón exclama Lucrecio: «Mortalem vitam mors cum immortalis ademit» (cuando la muerte inmortal ha extinguido la vida mortal)"'27. La dinámica contingente lucreciana de la materialidad -muerte, meteorito, ...-vuelven, para Marx, sin Aufhebung especulativa.

Rodeados por esta niebla de la guerra (fog of war) se nos dificulta poder ver con claridad quién termina sobreviviendo a la crítica despiadada del joven Marx, como él mismo escribió a Arnold Ruge en esos años. De todos modos, el supuesto "inútil" de Lucrecio ha sido bastante útil para este joven Marx. Para Fenves, no se puede determinar con precisión qué es lo que nos indicaría la tesis del concepto de ciencia que Marx afirma. Lo que

\footnotetext{
J. Lezra, On the Nature of Marx's Things, op. cit., p. 42.

25 P. Fenves, "Marx's Thesis on Two Greek Atomists and the Post-Kantian Interpretations", op. cit., p. 443

26 V. Morfino, El Tiempo de la Multitud, trad. Roberto Peña León, Mariana Gainza y Sebastián Torres, Santiago de Chile, Doble Ciencia Editorial, 2015, p. 42

27 K. Marx, Diferencia de la filosofía de la naturaleza en Demócrito y en Epicuro, op. cit., p. 19.
} 
sí queda claro es la búsqueda que lleva a cabo Marx de un concepto de ciencia -en un campo de batalla donde se encuentra con Kant y Hegel- y cómo tal búsqueda se relaciona con la desviación lucreciana.

Lezra cita un fragmento de los Cuadernos en el que Marx parece confirmar la lectura de Epicuro que acabamos de esbozar por medio de Fenves. En este fragmento Marx "describe el principio de movimiento atómico" en De rerum natura,

As the atom swerves away from its premise [Wie das Atom von seiner Voraussetzung ausbeugt], divests itself of its qualitative nature and therein shows that this divestment, this premiseless, contentless being-enclosed-in-self exists for itself, that thus its proper quality appears, so also the whole of the Epicurean philosophy swerves away from the premises; so pleasure, for example, is the swerving away from pain [Schmerz], consequently from the condition in which the atom appears as differentiated, as existing, burdened with non-being and premises. But the fact that pain exists, etc., that these premises from which it swerves away exist for the individual -this is its finiteness [seine Endlichkeit], and therein it is accidental... One swerves away from determinism by elevating [erhoben] accident, necessity and arbitrariness to the level of the Law; God swerves away from the world, it is not for him, and therein he is $\operatorname{God}^{28}$.

Para Marx, Epicuro parece querer escaparse del dolor ("pain [Schmerz]") de los átomos -podríamos decir, del retorno de tal exterioridad, de esa materialidad que lo acecha, esa materia reprimida que retorna en la muerte y los meteoritos: lo que Lezra identifica como la condición de Qual de toda materia, su tormento, por la que se desvía aleatoriamente. El Qual como contingencia de la materialidad ${ }^{29}$.

Es este lenguaje lo que parece autorizar la lectura de Lezra en la que Marx se desvía del nivel onto-epistemológico a un nivel ético »pero, ¿se podría marcar una diferencia tan fuerte entre estos dos niveles en el texto de Marx, más allá de pensar la autonomía relativa de ellos? Recordemos, como plantea Fenves, que una batalla clave en la tesis de Marx fue la de pensar un concepto de ciencia. Parece difícil que en un pensador que hereda a cierto spinozismo pueda mutilar el vínculo entre lo onto-epistemológico y lo ético. Más allá de encontrar una forma estética y una ética, ¿puede ser que Marx también encuentre en Lucrecio los elementos para pensar algo así como una ciencia de la contingencia a pesar de la ciencia de Kant y Hegel?

\section{Ciencia}

Siguiendo al Marx que emerge en el trabajo de Lezra nos encontramos con la subterránea ciencia de la contin-

\footnotetext{
J. Lezra, On the Nature of Marx's Things, op. cit., p. 44.

29 "Qual is the condition under which undifferentiated matter swerves, at uncertain times and in uncertain places, into differential relation, forming organized bodies; and it is the condition under which merely arithmetic relations «jump» into a qualitative relation, revealing an always-already operating law of measure retroactively". J. Lezra, On the Nature of Marx's Things, op. cit., p. 47.
}

gencia. En la última oración de la cita de Lezra podemos leer que el desvío del "determinismo" pasa precisamente por elevar el clinamen -llámese "accidente", pero también "necesidad", dice Marx- al nivel de la Ley ¿No es este el concepto de tendencia con el que trabaja Marx en El Capital? Por ejemplo, en la "ley de la tendencia decreciente de la tasa de ganancia", del tercer tomo, que constituye un pilar fundamental de la teoría de crisis marxiana. En una aproximación filosófica al concepto de tendencia en El capital, Balibar escribe que "[e] 1 carácter de tendencia aparece en principio como una carencia de la ley, pero una carencia extrínseca, causada por el obstáculo de circunstancias exteriores que no dependen de ella y cuyo origen es (por el momento) inexplicable" ${ }^{\prime 30}$. Por lo tanto, el concepto de Marx de tendencia, ahora difractado por Lucrecio y por cierta lectura de Lezra, se puede leer a la par de la ciencia de aquella desviación, de aquella contingencia radical de la "carencia extrínseca" -exterioridad o la materialidad que hace imposible (por ahora) su explicación pero que, al mismo tiempo, es conocible ${ }^{31}$.

¿Pero, por qué ciencia? ¿No está pasado de moda hablar de ciencia hoy? Siendo fiel al ejercicio crítico-político que convoca el trabajo de Lezra, nos parece urgente enfrentarnos al problema de la ciencia en una coyuntura en la que el capitaloceno y sus catástrofes -entre tantas otras, la de Covid-19 y la crisis climática- acechan la lucha tanto en la práctica política como en la práctica teórica. El giro que enfatiza Lezra en Marx hacía una ética -pero no en el sentido spinoziano, debido a que Spinoza no se deshace la imbricación con la dimension onto-epistemológica- parece ser sintomático. Si es Lucrecio el nombre de la desviación por la cual emerge el pensamiento de Marx, ¿cómo hacernos cargo del problema al que éste se aproximaba en su tesis y que parece tan crucial hoy? Por momentos parece que tal concepto de ciencia en la lectura de Lezra quedara desplazado por una lectura literaria debido a que en el tiende a identificar a la ciencia con una concepción estrictamente disciplinaria que desemboca en una abstracción debilitan$\mathrm{te}^{32}$. Tal lectura literaria, lejos de ser una noción culturalista, está vinculado con la traducción y lo que Lezra llama la necrofilología. Usando el léxico lezraeno, para Marx, la relación entre objetos de "primer orden", -conceptuales- y objetos de "segundo orden", -materiales- consiste en una mediación, un tercer objeto: objetos relacionales o "translational objects" 33 . En contraste con cierta "filología positiva", la necrofilología en Marx no produce una positividad. Tal sistema conceptual marxiano Lezra lo denomina como un sistema defectivo (defective). Veremos cómo en tal "sistema conceptual defectivo" emerge un concepto de ciencia.

$30 \quad$ L. Althusser, Para leer El Capital, trad. M. Harnecker, México, Siglo XXI, 2004, p. 311.

31 En una nota a pie de página, Lezra cita a Adorno e indica que en tal cita podemos encontrar una aproximación a un concepto adorniano de "tendencia" que se aproxima a lo que venimos trabajando aquí. J. Lezra, On the Nature of Marx's Things, op. cit., p. 233.

32 Aunque no queda desplazada la implícita ciencia melancólica adorniana, o, podría ser, La gaya ciencia que apunta hacia la "transvaloración de todos los valores".

33 Cf. J. Lezra, On the Nature of Marx's Things, op. cit., p. 21. Notemos que "translational" remite al significado de la palabra de traducción en inglés pero también al desplazamiento. 
Consideramos que hoy, en esta coyuntura, elaborar un concepto de ciencia se nos presenta como un campo de batalla crucial que, al mismo tiempo, no se le puede ceder a las "disciplinas". Pensamos que el desvío por Lucrecio que nos obsequia la lectura de Lezra, nos brinda una suerte de paralaje para leer en Marx otro concepto de ciencia -ni determinista ni disciplinario- que puede potenciar ciertas alianzas fortalecedoras ${ }^{34}$.

Siguiendo un criterio mínimo de cientificidad -el de la delimitación de sus objetos de estudio- nos podríamos preguntar, ¿qué tipos de objetos produce El capital? Seguramente esta es una pregunta poco "novedosa", pero toma otra consistencia luego de haber difractado a Marx por Lucrecio y sus declinaciones, sus objetos dinámicos y contingentes, como los llama Lezra, que fascinan al joven pensador. Para Lezra, como recién mencionábamos, los "objetos" de Marx no corresponden a ningún sistema disciplinario: "Capital produces dynamic conceptual objects unsuitable to the order and to the time of any decision-system or discipline -including the decision-systems that Capital itself produces" 35 . Es decir, más allá de que en El capital la maquinaria marxiana relaciona-traduce "objetos mentales" a "objetos materiales" por una mediación -un "tercer objeto"- para Lezra tal "sistema de conceptos" de Marx es defectivo. Tal sistema no nos remite a ninguna unidad o identidad disciplinaria -aunque tales objetos, como indica Lezra, si son expropiados por Marx tanto de la filosofía como también de las ciencias naturales, las matemáticas, la política y la religión. Lo que tenemos aquí es un problema de transformación. Aunque ya no en el sentido clásico de la economía política marxiana -a saber, el "problema de transformación" entre precios y valores-pero tampoco ajeno a él. Tal problema concierne una teoría de traducción de los objetos de Marx, siempre contingentemente dinámicos, sin cercarlos (en el sentido de "enclosure") en un sistemadisciplina. Pero, al mismo tiempo, reconoce Lezra, no obstante no es rastreable $u n$ sistema conceptual en El capital si existe una tendencia, una determinación, quizás en la última instancia o, para decirlo en términos matemáticos: una aproximación asintótica, que tiende hacia la sistematicidad (aunque siempre defectiva-dinámica). Tal "sistematicidad defectiva" se aproxima a lo que hemos llamado ciencia de la contingencia.

Lezra encuentra la "alternativa" de Marx al "enclosure" disciplinario en la famosa "Introducción de 1857". Es decir, es en la excavación del nombre propio de Spinoza, en $s u$ frase, "Determinatio est negatio", que irrumpe en la introducción metodológica del ' 57 , donde Lezra afirma que Marx desestabiliza la identificación de la "producción" y el "consumo" de los economistas políticos burgueses y, por lo tanto, desestabiliza también su método ${ }^{36}$. Por lo tanto, no es solamente en Lucrecio,

34 Siguiendo la lectura de la ética spinoziana, en la cual el efecto de una composición entre una idea con otra idea o un cuerpo con otro cuerpo necesariamente implica un incremento de la potencia.

35 J. Lezra, On the Nature of Marx's Things, op. cit., p. 59.

36 No podemos aquí indagar sobre esta relación, pero en esta proposición encontramos un Spinoza difractado por Hegel, difractado por Marx. La difracción divide, multiplica. Una diferencia en esa difracción-que Lezra no menciona- parece ser clave. Marx: Determinatio est negatio. Hegel: Omni determinatio est negatio. La formulación hegeliana es universalizada. La diferencia, por lo tanto, es literal- sino también en Spinoza, donde Lezra encuentra la contingencia dinámica de los objetos de Marx. Pero, ¿no es la "Introducción de 1857" donde Marx precisamente esboza también su "método científico", que el mismo contrasta con las robinsonadas de los economistas políticos burgueses? Nuestra insistencia en torno al concepto de ciencia se debe a que este incide también en la lectura de Marx y sus "cosas", concepto que él mismo buscaba en su tesis. En este punto buscamos "suplementar" la lectura de Lezra.

Retomando el hilo de On the Nature of Marx's Things, parte de la consistencia de la lectura lezreana de los objetos dinámicos y contingentes pasa por desestabilizar la "forma clásica" de una disciplina para entonces establecer un "puente" entre tres "registros" en Marx:

a classically scientific, even positivist register (a hypothesis or a cluster of concepts is tested against the facts of the case); an activist register (put these "unsuitable" objects in place, as descriptions or as regulative principles, and assess the outcome; violence is entailed in the first moment); an aesthetic, even gastronomic register (what formal principles, or principles of taste, are invoked, challenged, or suitably employed, when this "unsuitable" object is made to cover that unexpected body of facts and circumstances? $)^{37}$.

Por lo tanto, nuestra insistencia en la ciencia de la contingencia pasaría como suplemento del primer registro -el "clásicamente científico"- que, a contrapelo de cierto positivismo, podríamos encontrar en Marx como un concepto de ciencia que se aproxima a lo que Lezra llamaría lo necrológico -el mismo que ya hemos esbozado con el retorno de la exterioridad de los "meteoritos" y la "muerte" en la tesis de Marx. ¿Cómo releer entonces los elementos científicos en Marx al repensarlos como objetos dinámicos? ¿Cómo leer sus citas constantes de las ciencias de su tiempo? Por ejemplo, de la biología: la célula-commodity (mercancía) y la ciencia contingente de la evolución (darwiniana). $\mathrm{O}$ también, a la física del siglo XIX, la termodinámica que fascinaba también a Freud y la "segunda ley", que define el concepto de entropía. Dos conceptos -el de termodinámica y el de entropía- a los que el mismo Lezra recurre para "definir" la lectura "literaria" de Marx, su necrofilología ${ }^{38}$.

En segundo lugar, nuestra insistencia en volver a indagar en torno al concepto de ciencia de Marx pasa también por cierta lectura de nuestra coyuntura -en la cual la ciencia o, en este caso, las ciencias "naturales" son

mente todo (omni). ¿Puede ser que para Marx haya determinaciones que no sean negaciones?

37 Ibidem, pp. 59-60. Resaltado propio.

38 "We might instead say that reading for things after Marx, reading necrophilologically, means attending to the way in which Marx's (and others') reflections on physical and conceptual objects translate into reflections on the form of statements regarding concepts. To read necrophilologically is to read with an eye to how texts both 'reflect' and 'break' the drive to the object. It means to engage arguments not as formal, tendentially organic, autopoetic factories of 'life,' as philosophies or philologies of life, but as partial drives, as dynamics, as polemics, as devices for producing deformed forms, engaged in violent practices of translation: as a thermodynamics. Every argument about objects after Marx is literary in this quite specific way". Ibidem, pp. 10-11. 
reducidas a un "instrumento de gobierno". En este sentido el reciente caso de Agamben resulta paradigmático.

En un corto ensayo titulado ¿Qué es real? el filósofo italiano Giorgio Agamben indaga en torno a la misteriosa desaparición del famoso físico italiano Ettore Majorana una noche de marzo del año 1938. Majorana, uno de los físicos más importantes de su generación, quien desarrolló elementos claves de la física cuántica de esos años, le provee a Agamben un trasfondo dramático donde situar la problemática relación entre la ciencia y "lo real". Hasta el día de hoy no se sabe bien qué pasó con Majorana ¿Por qué desapareció? Esta incertidumbre da lugar a la interpretación especulativa del destino del joven físico italiano. ¿Se suicidó? ¿Se escapó porque ya no soportaba el rumbo hacia donde se dirigía la ciencia? No es este el lugar para desarrollar el argumento de Agamben en todas sus aristas, pero cabe decir que el filósofo italiano enfoca sus meditaciones en un corto ensayo que escribió Majorana, publicado un año antes de su desaparición y titulado "El valor de las leyes estadísticas en la física y en las ciencias sociales", para plantear, siguiendo el pensamiento analógico del físico, que la emergencia de las leyes estadísticas en las ciencias da paso al "arte del gobierno". Es decir, el supuesto derrocamiento del determinismo de la física clásica por la mecánica cuántica y sus principios de "incertidumbre" o "contingencia", "autoriza" y "permite" al investigador "comandar" o "determinar" el sistema en cuestión. En la contingencia, Agamben -por medio de la analogía de Majorana- encuentra nada más que el gobierno. La ciencia, en particular "la ciencia moderna," dice Agamben, da paso directamente al gobierno de las $\operatorname{cosas}^{39}$. En tal lectura, la materialidad -o "lo real"- pareciera no tener su "externalidad" (como el meteoro y la muerte en la Diferencia de Marx). Como si lo "real" fuese determinado unilateralmente por la arbitrariedad soberana del investigador. En tal lectura, la ciencia de la contingencia no es más que gobierno.

A contrapelo de tal lectura que encierra a la ciencia en lo disciplinario - es decir, en el "arte del gobierno"- la ciencia de la contingencia que nos concierne se podría pensar como una ciencia menor, una ciencia nómade, tal como la describen Deleuze y Guattari en Mil Mesetas,

Existe un tipo de ciencia, o un tratamiento de la ciencia, difícilmente clasificable, y cuya historia tampoco es fácil de seguir. No son "técnicas", según la acepción habitual. Tampoco son "ciencias", en el sentido real o legal establecido por la historia. Según un reciente libro de Michel Serres, se puede rastrear su huella en la física atómica, de Demócrito a Lucrecio... ${ }^{40}$.

La herencia lucreciana de esta ciencia nómade no es casual. Deleuze y Guattari la identifican como una ciencia aguerrida: "[e]s como si el «científico» de la ciencia nómada estuviera atrapado entre dos fuegos, el de

\footnotetext{
39 "La scienza moderna - e, con essa, ogni singolo uomo - orienta le sue decisioni secondo un criterio che non può riferirsi direttamente al caso in questione, ma solo a un «caso probabile» che non coincide se non «casualmente» con quello". G. Agamben, Che cos'è reale? La scomparsa di Majorana, Vicenza, Neri Pozza Editora, 2016, pp. 40-41.

40 G. Deleuze, y F. Guattari, Mil mesetas, capitalismo y esquizofrenia, trad. J. Vázquez Pérez, Valencia, Pre-textos, 2002, p. 368.
}

la máquina de guerra que lo alimenta y lo inspira, el del Estado que le impone un orden de razones"41. Tal ciencia nómade de la contingencia - de herencia lucreciana- no es ni reducible a la razón disciplinaria, ni al gobierno de las cosas ¿No es este el concepto de ciencia que encontramos en el joven Marx de la Diferencia como también en el concepto de tendencia en El capital?

Por último, el contraste con la noción de la ciencia y de la contingencia que encontramos en Agamben y la ciencia de la contingencia que encontramos en Marx pasa por pensar la indeterminación de la relación entre los objetos del primer y según orden, tal como los piensa Marx. Es ahí donde su "thermodynamic drift", como le llama Lezra, su dinamismo, su contingencia, determina -es ahí donde se expresa la necesidad de la contingencia y la contingencia de la necesidad. El argumento analógico agambeniano desplaza tal posibilidad y por lo tanto afirma una lectura unilateral de la mediación, del entrelazamiento, clausurando con ello otra interpretación posible de la mecánica cuántica. Es justamente desde este concepto de entrelazamiento (entanglement) que Karen Barad elabora su materialismo performativo desde una lectura de la mecánica cuántica y los problemas que esta ciencia de la contingencia presenta para un materialismo vulgar y humanista.

\section{Alianzas}

En la introducción a su libro Meeting the Universe Halfway: Quantum physics and the Entanglement of Matter and Meaning, la pensadora feminista y física teórica Karen Barad hace un llamado a ser cautelosos con el pensamiento analógico que encontramos en ciertas interpretaciones de la mecánica cuántica. Allí, Barad sitúa la escena del encuentro entre Werner Heisenberg y Niels Bohr en septiembre de 1941 en la capital danesa. Los dos físicos son artífices de la "Interpretación de Copenhague" de la mecánica cuántica que articula el principio de complementariedad de Bohr y el principio de incertidumbre de Heisenberg. La escena a la que acude Barad de tal encuentro corresponde a una obra de teatro del dramaturgo británico Michael Frayn que lleva el mismo nombre que la capital danesa -ciudad que en ese entonces estaba ocupada por tropas nazis ¿Por qué fue el joven físico aleman, Heisenberg, a encontrarse con su mentor de descendencia judía? Escribe Barad,

This question -why Heisenberg went to see Bohr in 1941- is the focal point of a recent Tony Award-winning play that considers the controversy surrounding this fateful meeting. The play doesn't resolve the controversy; on the contrary, the play itself has gotten caught up in its very orbit. In Michael Frayn's play Copenhagen, the ghosts of Bohr, Heisenberg, and Bohr's wife, Margrethe, meet at the old Bohr residence to try to reconcile the events of that fateful autumn day. As if working out the details of a problem in atomic physics, Bohr, Heisenberg, and Margrethe make three attempts to calculate Heisenberg's intentions, by enacting and at times stopping to reflect on three possible scenarios of what might have occurred. Each attempt to resolve the uncertainty is foiled. But that is precisely the point

$\overline{41 \quad \text { Ibidem, p. } 370 .}$ 
Frayn wishes to make: drawing an analogy with Heisenberg's uncertainty principle, Frayn suggests that the question of why Heisenberg came to Copenhagen in 1941 does not remain unresolved for any practical reason, such as some insufficiency in the historical record that can be straightened out with newfound evidence or some new clarifying insight, but rather is unresolvable in principle because uncertainty is an inherent feature of human thinking, and when all is said and done, no one, not even Heisenberg, understands why he came to Copenhagen ${ }^{42}$.

En la lectura de Barad - a contrapelo de la lectura de Frayn $\mathrm{y}$ de Agamben, que proceden por medio de un argumento analógico-pareciera como si la incertidumbre fuese un principio que aplicase indiscriminadamente a todo. El tema es que el argumento es tan grueso que no aplica estrictamente tampoco para los experimentos de la física. Para Barad, las propiedades de los fenómenos cuánticos (como la posición o el impulso), no son "gobernadas por el deseo o la voluntad" del investigador, pero si están determinadas por el aparato de medición ${ }^{43}$. Por lo tanto, lo que hay en juego es un entrelazamiento o un "assemblage" entre elementos heterogéneos que se determinan de un modo iterativo. Tal es el elemento central de cierto concepto de complementaridad de Bohr que recupera Barad en su lectura de la mecánica cuántica. Por lo tanto, para Barad, la determinación arbitraria y soberana del investigador no determina unilateralmente al "objeto dinámico" de la mecánica cuántica ${ }^{44}$. En un ensayo titulado "What is New Materialism?" se caracteriza al materialismo que Barad elabora desde los problemas que emergen de su interpretación de la mecánica cuántica como un materialismo performativo. Su singularidad -en contraste con los "nuevos materialismos" negativos, como el de Meillasoux- pasa por desplazar el excepcionalismo humanista de los otros dos ${ }^{45}$.
Volviendo al hilo del nombre de Marx en On the Nature of Marx's Things, nos encontramos, al final del libro de Lezra, con una suerte de deconstrucción de los "nuevos materialismos" que, para él, comparten una topología en común con el humanismo y con lo que llama "fetichismo teológico" $"$. Por lo tanto, en el complejo entramado de relaciones que Lezra va tejiendo en torno a Marx, Lucrecio, y Spinoza, entre otros, nos encontramos con una estrategia deconstructiva en la cual los "nuevos materialismos" humanistas se quedan pegados como en una telaraña. Pero como hemos rápidamente aquí esbozado, el único materialismo que parece poder escaparse de tal estrategia deconstructiva lezreana es el materialismo performativo de Barad. ¿En qué consiste entonces la diferencia del materialismo performativo de Barad? En, (1) la indeterminación constitutiva - la contingencia radical- de la "materia" ("pedetic"), (2) la primacía de la relación (aquellos que llamamos "entanglement" o entrelazamiento), y (3) el carácter iterativo de la interacción ${ }^{47}$. Al evocar el materialismo performativo de Barad, no pretendemos establecer una equivalencia entre el materialismo de Marx que hemos caracterizado - por medio de Lezra, y seguramente de un modo apresurado- como ciencia de la contingencia. Más allá de tal equivalencia, si afirmamos la posibilidad de nombrar la potencialidad de una alianza que espera ser elaborada. Alianza sin equivalencia que de todos modos podría potenciar un concepto de ciencia más allá de la enclosure disciplinaria. Como bien menciona el filósofo norteamericano Thomas Nail, el materialismo perfomativo de Barad hereda la corriente subterránea que lleva el nombre de Lucrecio y también el de Marx y sus $\operatorname{cosas}^{48}$.
42 K. Barad, Meeting the Universe Halfway: Quantum Physics and the Entanglement of Matter and Meaning, Durham, Duke University Press, 2007, p. 4. Resaltado propio.

43 "Which properties become determinate is not governed by the desires or will of the experimenter but rather by the specificity of the experimental apparatus. Thus there is still an important sense in which experiments can be said to be objective". Ibidem, p. 19.

44 Ibidem, p. 23.

45 "On one hand, negative new materialism embraces either a radical division between human thought and inorganic matter or a "withdrawn» essence, both of which we think persist due to its uncritical embrace of an external, human- observer perspective. On the other hand, while vital materialism explicitly rejects any form of essentialism, we think it nevertheless manages to sneak back in through a metaphysics of life projected onto inorganic matter. In these crucial ways, as we elaborate below, non-performative new materialist theories continue to implicate certain objectivist, non-relational and, thus, idealist assumptions or residuals". C. N. Gamble, Joshua S. Hanan y Thomas Nail, "What Is New Materialism?", Angelaki, 24, 6, 2019, pp. 11-134, DOI: 10.1080/0969725X.2019.1684704, p.112.
J. Lezra, On the Nature of Marx's Things, op. cit., pp. 184-5.

47 "Barad provides a particularly compelling basis for such a view through her «intractive» account of the «measurement problem» in quantum physics. This problem arose with the famous double-slit experiments in which, depending on the experimental arrangement, light (or atoms etc.) appears either as a wave or particle, despite their mutually exclusive properties. While debate continues to rage even today over how best to interpret these conflicting findings, its basic contours were largely defined by the early interpretations of Erwin Schrödinger, Werner Heisenberg, and Niels Bohr.... What Barad seizes on...is that entities simply do not determinately exist apart from the particular, physical measuring apparatuses that constitute them one way to the exclusion of others. Inherently, then, light, like all matter, is indeterminate. And thus, what light is, as a (relatively) determinate entity, does not entirely precede - and is not fully separable from - the physical, material apparatus used to observe it". Christopher N. Gamble, Joshua S. Hanan \& Thomas Nail, "What Is New Materialism", op. cit. p. 122.

48 Cf. T. Nail, Marx in Motion: A new materialist Marxism, Oxford, Oxford University Press, 2020. 


\section{Bibliografía}

Agamben, G., Che cos'è reale? La scomparsa di Majorana, Vicenza, Neri Pozza Editora, 2016.

Althusser, L., Para un materialismo aleatorio, Madrid, Arena Libros, 2002.

-, Para leer el Capital, trad. M. Harnecker, México, Siglo XXI, 2004.

Barad, K., Meeting the Universe Halfway: Quantum Physics and the Entanglement of Matter and Meaning, Durham, Duke University Press, 2007.

Derrida, J., Espectros de Marx, Madrid, Editorial Trotta, 1998.

Deleuze, G. y F. Guattari, Mil mesetas, capitalismo y esquizofrenia, trad. J. Vázquez Pérez, Valencia, Pre-textos, 2002.

Fenves, P., "Marx’s Thesis on Two Greek Atomists and the Post-Kantian Interpretations", Journal of the History of Ideas 47, 1986.

Gamble, C.N., Joshua S. Hanan y Thomas Nail, "What Is New Materialism?”, Angelaki, 24, 6, 2019, pp. 111-134, DOI: 10.1080/0969725X.2019.1684704.

Lezra, J., On the Nature of Marx's Things, New York, Fordham University Press, 2018.

Marx, K., Diferencia de la filosofia de la naturaleza en Demócrito y en Epicuro, Madrid, Editorial Ayuso, 1971.

Marx, K., Capital: A Critique of Political Economy, Vol. 1, Penguin Books, 1990.

-, A Critique of Political Economy, Vol. 3, London, Penguin Books, 1991.

-, Grundrisse: Foundations of the Critique of Political Economy, London, Penguin Books, 1993.

Morfino, V., El Tiempo de la Multitud, trad. Roberto Peña León, Mariana Gainza y Sebastián Torres, Santiago de Chile, Doble Ciencia Editorial, 2015.

Nail, T., Marx in Motion: A new materialist Marxism, Oxford, Oxford University Press, 2020.

Read, J., "Primitive Accumulation: The Aleatory Foundation of Capitalism", Rethinking Marxism, 14,2, 2002, pp. 24-49. DOI: $10.1080 / 089356902101242161$.

Villalobos-Ruminott, S., "La querella de las cosas: Marx, Lucrecio y el desorden del mundo", Oxímora Revista Internacional de Ética y Politica, 17, 2020, pp. 119-134, DOI:10.1344/oxi.2020.i17.31587. 